\title{
Guest editorial: new developments in future networked systems
}

\author{
Haroon Malik ${ }^{1}$ - Ansar-Ul-Haque Yasar $^{2}$ - Elhadi M. Shakshuki ${ }^{3}$
}

Published online: 23 June 2015

(c) Springer-Verlag Berlin Heidelberg 2015

This special issue is based on the best papers from the 5 th International Conference on Emerging Ubiquitous Systems and Pervasive Networks (EUSPN-2014), which was held in Halifax, Canada, on 22-25 September 2014. The conference attracted a large number of scientific papers that contributed to the state-of-the-art in the ground-breaking invention to future communication technologies, including mobile broadband and optical networks. All the papers selected for this special issue have been extended from their original versions and underwent three rounds of rigorous peer-review process. Based on the reviewers' feedback, as well as the evaluations of the Guest Editors, six papers were selected for this special issue from eight invited submissions. The accepted papers cover interesting works on new developments in future networked systems such as Internet of Things (IoT), pervasive information systems and optical infrastructure.

The first paper by Engel et al. is entitled "Performance improvement of data mining in Weka through multi-core and GPU acceleration: opportunities and pitfalls". This paper investigates strategies to improve the performance

Elhadi M. Shakshuki

elhadi.shakshuki@acadiau.ca

Haroon Malik

malikh@uwaterloo.ca

Ansar-Ul-Haque Yasar

ansar.yasar@uhasselt.be

1 School of Computer Science, University of Waterloo, Waterloo, Canada

2 Transportation Research Institute, Hasselt University, Hasselt, Belgium

3 School of Computer Science, Acadia University, Wolfville, Canada of Weka, a popular data-mining tool, through multi-core and GPU acceleration. Using java profilers, the authors identified a set of operations that are time-consuming and that can easily be adapted to multi-cores and GPUs; Thereby, improving the data mining performance when parallelized. Authors selected two operations, i.e., matrix multiplication and sort and parallelized them to take advantage of multi-core CPUS and GPUs. As a result, the authors observed speedup levels of at least 1.49 with respect to the current Weak distribution, drastically decreasing the time the algorithm consumes to analyse a dataset.

The second paper by Helmy et al. is entitled "Semantic manipulation of user's queries and modelling the health and nutrition preferences". The paper harness semantic web technology to capture user's preferences, construct a nutritional and health-oriented user's profile, model the user's preferences and use them to organize the related knowledge so that users can retrieve personalized health and food information. In particular, authors have proposed an agent-based system for semantic query manipulation and results personalization. They modelled the processes of semantic query manipulation and personalization and presented how we capture the user's preferences and create the user's profile-based ontology.

The third paper by Najar et al. is entitled "Service discovery and prediction on pervasive information systems". The authors presented a new user-centric approach, for the pervasive information systems (PIS) in addition to a new service discovery and prediction based on both user's context and intentions. The intentions not only allow context, which influence the service implementation but also to anticipate the future user's needs in an understandable way. In addition, the authors proposed a service discovery mechanism that observe user's context and 
intention in order to offer him/her the most appropriate service satisfying on the current context.

The fourth paper by Jaskolka et al. is entitled "Investigative support for information confidentiality". The authors have proposed an algebraic technique using relations for detecting confidential information leakage via protocol-based covert channels. Covert channels can be used to transmit sensitive information in a secret manner. This makes them a particular threat to the confidentiality of information. The proposed technique does not rely on heuristics to uncover the use of covert channels. Indeed, provide a formal and rigorous approach that offers a degree of simplicity. Authors also presented an application of the proposed technique in the area of cryptanalysis. The presented cryptanalysis technique uses the relational representation of information and the tests to verify the existence of an abstraction relation and computations, to find the abstraction relation, if it exists from the technique, for detecting confidential information leakage in conjunction with notions from known-plaintext attacks to aid in uncovering plaintext messages from a given cipher text. Authors also pointed to the development of tools to aid in the automation of the proposed technique and its applications.

The fifth paper is by Jararweh et al. is entitled "SDIoT: a software defined based internet of things frame work". Internet of Things (IoT) is a vision. It is being built today. The authors have built a framework for Software Defined Internet of Things (SDIoT) that exploits several softwaredefined systems such as software-defined network, software defined store and software defined security. The authors first talk about their proposed SDIoT architecture model and then show how they exploited the ideas from software-defined system to build it. In the paper, they provide in detail the main elements of SDIoT. Authors also showed how these elements interact with each other to provide a comprehensive framework to control the IoT network.

The sixth paper by Nafarieh et al. is entitled "A comprehensive analysis of QoS-based routing mechanisms over shared mesh protected optical infrastructures". The paper is a literature overview of the service level agreement (SLA) based architecture over priority-aware shared mesh optical networks. The fundamental module of the discussed architecture has been introduced as the SLA negotiation infrastructure. The standardized protocols and existing algorithms for path attributes and SLA parameters negotiation have been studied in this paper and shortcomings of each have been identified. A thorough study of the priorityaware, SLA-based, and risk-are mechanisms including the existing mechanisms, their advantages and disadvantages, and the remedy for the shortcomings has been provided. The mathematical models for availability analysis of different routing mechanisms, protection schemes, and network resources have been discussed. Different routing strategies and wavelength assignment approaches have been suggested.

The guest editors would like to take this opportunity to thank all the authors for the efforts they put in the preparation of their manuscripts and for their valuable contributions. We wish to express our deepest gratitude to the referees who provided very useful and thoughtful feedback to our authors. Our sincere thanks go to the Editor-in-Chief for his kind help and support. 